\title{
¿Hacia la autonomía educativa? La 4T y la crisis educativa en México
}

Towards educational autonomy? 4T and the educational crisis in Mexico.

Em direção à autonomia educacional? 4T e a crise educacional no México.

\author{
Martha Areli Ramírez Sánchez \\ areliramirez31@yahoo.com.mx \\ Profesor investigador Universidad Iberoamericana - México \\ https://orcid.org/0000-0002-7858-2915
}

\begin{abstract}
RESUMEN
La crisis educativa en México agudizada por la Covid 19, solo dejó en evidencia problema añejos irresueltos en todo el sistema educativo, resultado de procesos de privatización sobre todo en los niveles de educación media y superior. Pero desde el 1 de diciembre del 2018 con la llegada de la izquierda histórica al poder, miembros de organizaciones afines que han desarrollado modelos de educación alternativa, esperaron finalmente poder participar en la transformación y solución de estos problemas. Sin embargo, las políticas en materia educativa del actual gobierno, autonombrado la 4T, dio la espalda a estas iniciativas, que de facto y desde hace 3 décadas, promueven y proponen soluciones viables pasa resolver dicha crisis. Ante la negativa de otorgárseles el reconocimiento oficial se han dado a la tarea de agruparse en red de redes creando sus propias instancias de autorreconocimiento, mostrando vitalidad, creatividad y resistencia ante los diversos cambios de gobierno caminando entonces hacia la autonomía educativa.
\end{abstract}

Palabras clave: Crisis educativa, autonomía educativa, $4 \mathrm{~T}$

\begin{abstract}
Mexican educational crisis exacerbated by covid 19, only left unresolved old problems in evidence throughout all the educational system, as a result of privatization processes in former governments, especially at secondary and higher education. However, since December 1, 2018, with the arrival of the historic left to power, members of like-minded organizations that have developed alternative education models, have finally hoped to be able to participate in the transformation and solution of these problems. However, the educational policies of the current government, self-appointed as the 4T, turned its back on these initiatives, which de facto and for 3 decades, promote and propose viable solutions to solve the crisis. Faced with the refusal to grant them official recognition, they have taken on the task of grouping themselves into a network of networks creating their own instances of self-recognition, showing vitality, creativity and resistance to the various changes of government, always moving towards educational autonomy.
\end{abstract}

Key words: Educational Autonomy, educational crisis, $4 \mathrm{~T}$

\section{RESUMO}

A crise educacional no México, exacerbada por covid 19, só deixou velhos problemas não resolvidos em evidência em todo o sistema educacional, resultado de processos de privatização, especialmente nos níveis de ensino médio e superior. Mas desde $1^{\circ}$ de dezembro de 2018, com a chegada da esquerda histórica ao poder, membros de organizações com ideias semelhantes que desenvolveram modelos de educação alternativos, finalmente esperam poder participar da transformação e solução desses problemas. Porém, as políticas educacionais do atual governo, autoproclamado 4T, deram as costas a essas iniciativas, que de fato e há 3 décadas, promovem e propõem soluções viáveis para a solução da crise. Diante da recusa de reconhecimento oficial, assumiram a tarefa de se agrupar em uma rede de redes criando instâncias próprias de autoreconhecimento, demonstrando vitalidade, criatividade e resistência às várias mudanças de governo, caminhando então para a educação. autonomia.

Palavras chave: Crise educacional, autonomia educacional, $4 \mathrm{~T}$ 


\section{INTRODUCCIÓN}

Como salida a la emergencia educativa que se vive en México, agudizada por la pandemia de la COVID -19, en este documento exponemos alternativas educativas creadas por comunidades de base. Iniciativas que han generado espacios de autonomía educativa y han probado modelos que funcionan aún en tiempos de emergencia, gestionando sus propios sistemas de aprendizaje, admisión y evaluación. Los cuales, sin embargo, se ven invisibilizados por el Estado en sus continuos esfuerzos por centralizar e institucionalizar toda experiencia educativa en el país. En palabras de Hanna Arendt (1959) la educación se convirtió en una cuestión política. Y no precisamente en el presente sexenio, históricamente la universidad fue el vehículo para la creación de la clase política (Mendoza Rojas, 2001). Obra comparable con la de Fray Pedro de Gante y la creación de la Escuela de San José de Belén de los Naturales en 1522 y posteriormente El Colegio de Texcoco en 1525, escuelas para la desindianización de los naturales (López de la Torre, 2015). Han pasado ya muchos siglos de estos eventos sin embargo pareciera que las lógicas que la subyacen continúan.

Son ya un poco más de tres años que el sistema de partidos dio un giro inesperado al ganar la izquierda institucionalizada los comicios del 2018 en México. Por primera vez un candidato de la izquierda histórica ha ganado la presidencia, en los primeros momentos se pensó en un panorama diferente para sus aliados los movimientos populares. No obstante, para las escuelas autogestivas la situación no parece mejorar en nada, al contrario, se han visto excluidas ante las nuevas acciones del gobierno en esta materia.

Para ahondar en las contradicciones que la nueva administración ha generado seleccionaremos 3 esferas del amplísimo espectro de la educación en México. Primeramente, la emergencia educativa, como resultado de un cúmulo de problemáticas no resueltas en las últimas décadas por los diversos gobiernos. En segundo lugar, ubicaremos dicha crisis en el contexto de la Cuarta Transformación, de aquí en adelante nos referiremos a esta como la 4T. Y finalmente abordaremos los sistemas de educación de nivel medio y superior autogestivos, experiencias que por su propio origen son tan diversas como la cultural misma del país.

En este documento ponemos en el centro de la discusión un grupo de iniciativas, que configuran de manera general experiencias de un espectro político tan variado que sería necesario un capitulo para cada una de ellas. Ejercicios etnográficos profundos, que analicen la historia, estructura organizativa, propuesta pedagógica y andragógica, su impacto en la comunidad y postura política, entre otras cosas. La complejidad de sus historias políticas los une y los separa del sistema de partidos, de la política 
institucionalizada, algunos otros se mantienen al margen. Otros más afirman ser autogestivos pero reciben o buscan el apoyo de algún actor político local institucional, pero su sustento principal surge de sus comunidades de la cual dependen. Su vibrante actividad política es tan intensa como la vida política de América Latina (Peralta, 2019; Cristi, 20018; Aguirre, 2017, García, 20015; Zibechi, 2014; Batta, 2008).

\section{El giro hacia la izquierda}

El 4 de julio del 2018 en México se realizaron los comicios para elegir presidente y renovar las cámaras de senadores y de diputados. Se renovaron 629 cargos incluyendo al jefe del ejecutivo, se vivió una jornada electoral sin precedentes, por primera vez ganó la presidencia un candidato de la vieja izquierda. El heredero de la carrera política labrada desde los de movimientos de la izquierda popular, campesina, vecinal, gestada desde 1960.

El resultado sorprendió a muchos y a pie de calle se podía ver, para los que seguimos la jornada directamente, una sorpresa y esperanza de una transformación efectiva no solo en la administración pública del país, sino una oportunidad de participar en esa vida política que hasta antes se había negado. A 3 años y un poco más del medio término de esta administración, no es claro el futuro de muchos de los que impulsaron la campaña de la que entonces se llamó la coalición “Juntos Haremos Historia". Decisiones en materia educativa han confrontado a la 4T con sus antiguos aliados, lo cierto es que las alternativas educativas que aquí veremos interpelan al gobierno y al estado, al mostrar su osadía de existir en tiempos de emergencia educativa, desafiando las lógicas institucionales estatales.

Es tal su variedad que en este espacio aspiramos a comunicar un mundo de excepciones, una red de redes que puede extender los alcances reales del acto de educar, si y solo si, la comunidad se hace cargo de sus propios procesos educativos, administrativos y de sus jóvenes. En este momento uno de sus rasgos más importantes es que enfrentan a un contrincante muy fuerte, el estado y sus aparatos de gobierno; el cual, ante su insistencia de centralizar y gubernamentalizar todas las iniciativas educativas, ahoga los intentos de autonomía comunitaria.

\section{Notas sobre la educación superior en México.}

Renate (2006) en su trabajo señala algunas etapas de la transformación de la Universidad Nacional en México desde su fundación en el siglo XVI. Por ejemplo, su proceso de secularización 
total en 1910, donde nace como la Universidad Nacional Autónoma de México. La obtención de su autonomía relativa en 1929 y hasta su expansión ilimitada entre 1955 y 1960 (Rojas, 2015; Rodríguez, 2015). De tal manera que, en solo 7 décadas, desde la fundación del estado post-revolucionario se perfiló como un vehículo de ascenso y movilización social, compitiendo con la condición de raza (Viafara,popula 2017). Ese imaginario perduró hasta la gran huelga estudiantil de 1999 (Najera, 2020). Si bien toda institución debe adaptarse a los cambios históricos para sobrevivir, esta universidad evoluciono de la mano del Estado, adjudicándose la tarea de construir a la persona que el Estado Postrevolucionario quería.

El estallido zapatista de 1994 en Chiapas, reavivo e impulso colectivos y organizaciones que entre otras cosas cuestionaron la función de la universidad. De tal manera que al termino de los 90, se vivieron años muy agitados en términos educativos. Por ejemplo, en 1996 se creó el Movimiento de Aspirantes Excluidos de la Educación Superior (MAES). En el cual se agruparon aspirantes rechazados de la UNAM, que no lograron el ingreso después de presentar el examen de admisión. Se le sumaron rechazados de la Universidad Autónoma Metropolitana (UAM) y del Instituto Politécnico Nacional (IPN), con la demanda de obtener el ingreso en alguna universidad pública. Hasta el conflicto más grande, la huelga de la UNAM que duró 10 meses, el conflicto reavivo el debate sobre lo que es y debe ser la educación superior (Rodríguez, 2000). Finalmente, en la Universidad de la Ciudad de México UACM, en abril del 2011 se suscitó otro conflicto que pararía la Universidad por 90 días (animal político 2012).

Aunque estas movilizaciones tuvieron un impacto trascendental en la historia del país, reflejo solo una dimensión de lo que enfrentan millones de jóvenes mexicanos en edad de estudiar una carrera universitaria. Hay millones que no tienen siquiera la oportunidad de pelear, los mismos que se ven descartados y marginados de los procesos de ingreso y permanencia en estas casas de estudio. Hace 6 años, por ejemplo, la Dirección General de Administración Escolar, reportó que de los 60 mil 254 estudiantes que presentaron examen de admisión a la UNAM, para cursar alguna de las 113 licenciaturas que se ofrecen en modalidad escolarizada, abierta y a distancia, sólo 6 mil 893 fueron admitidos, es decir, solo el $11.4 \%$ de solicitantes fueron aceptados.

De acuerdo con Guzmán (2012) los mecanismos de ingreso a la educación superior "ponen al descubierto mecanismos de selectividad social. Se ha encontrado que los aspirantes que cuentan con un nivel socioeconómico más alto, tienen mayores probabilidades de ingresar a la UNAM" (2012: 134). Es un secreto a voces que para aumentar tus posibilidades de ingreso en el registro de examen debes 
incluir una dirección de residencia permanente ubicada en la Ciudad de México. Un análisis profundo de las condicionantes que derivan en una exclusión masiva de población joven en edad de cursas una carrera del sistema de educación superior, nos obligaría a incluir las variables clase, raza y etnicidad. Desafortunadamente esta es una tarea pendiente, de la que nos encargamos en este momento.

Para ilustrar la situación de otro sector aún más marginado de la educación superior el Censo de Población y Vivienda del 2010 (INEGI) señaló que de la población indígena de entre 15 y 24 años, solo el $62 \%$ completó la educación básica. De los cuales sólo el 3\% tuvo acceso a la educación superior. Las cifras actuales son aún más desalentadoras, desde el inicio del sexenio de Felipe Calderón (20062012) y su estrategia militar llamada "Guerra contra el narco"; un altísimo porcentaje de mujeres y hombres en edad de estudiar una carrera, perdieron la vida o paradójicamente se incorporaron al narcotráfico (Rodríguez, 2015; Ornelas, 2012). Violencia, pobreza, origen étnico, sexo, género, globalización son algunas de las variables mínimas a consideran como causa y efecto de la exclusión escolar, importantes trabajos se han desarrollado al respecto, arrojando datos y análisis profundos, que permiten armar un rompecabezas de esta complicada problemática (Saravi, 2015; Mingo, 2010).

\section{FUNDAMENTO TEÓRICO}

The United Nations Office for the Coordination of Humanitarian Affairs (OCHA, 2013), presenta el programa Educación en Emergencia como concepto y acción que busca proveer a la niña y niño de recursos materiales y emocionales para garantizarles una experiencia escolar. Uniendo la educación formal e informal, tradicional e institucional se persigue el objetivo de proteger a la infancia en medio de conflictos armados o desastres naturales. OCHA coordina las acciones entre organismos internacionales, gobiernos nacionales y actores locales.

La emergencia sanitaria en la que el mundo está envuelto desde los primeros meses del año 2020, expuso para propios y extraños los limites y deficiencias de los sistemas educativos. Tales como, la falta de un plan de emergencia y hasta la carencia de los medios más básicos para comunicar a directivos con sus profesores y alumnos. Si bien los estragos que la COVID-19 causo fueron diferenciados, cuantitativamente sus efectos son devastadores. En estos últimos meses del año 2021 aun no podemos evaluar los daños. Pero podemos afirmar que las instituciones de educación privada sortearon mejor la crisis, lo que aumento la brecha aún más entre escuelas públicas y privadas. Así como los jóvenes que se pueden mantener en la modalidad a distancia y los que desertaron. 
No está de más decir que la actual crisis sanitaria, impuso más presión para los países que ya instauraban medidas para educar en emergencia, por ejemplo, los países con conflictos armados generalizados (Humanitarian Response, 2021). De acuerdo con la ONG Entre Culturas, la cual depende de la compañía de Jesús, el concepto mismo se ha ido transformando, a medida que las emergencias se han ido diversificando o incluso las crisis se han vuelto crónicas. La emergencia se han vuelto la normalidad (Vera e Icaza, 2015).

A pesar de la gravedad de esta crisis solo el rector del Centro de Estudios Macroeconómicos de Argentina, UCEMA pidió declarar una emergencia educativa, al comprender los enormes retos que implica la educación a distancia (Zablotsky, 2020). Desde pobre conectividad a internet, la falta de una computadora en casa, falta de internet en casa, teléfonos celulares obsoletos que no alojan las aplicaciones necesarias para conectarse a las clases, o que los alumnos no disponen de una de teléfono celular. De acuerdo con la revista Forbes hacían junio del año 2021, el aumento porcentual de usuarios de internet en México llego al 72\%. Lo que representa 84.1 millones de usuarios, sin embargo, esto no significa que sepamos usar las herramientas tecnológicas, con fines educativos de manera adecuada (Miranda y Delgado, 2021). Pero ya sea que la educación en emergencia, sea vista como un acto humanitario, una necesidad o un derecho, aun depende de la buena voluntad de los gobiernos, de los organismos internacionales y actores sociales que tienen influencia directa en la toma de decisiones. Esta crítica no es novedosa, De la Torre (20017) entre muchos otros, han señalado tempranamente que las reformas estructurales impuestas desde el 2015 comenzaron a fracturar el sistema educativo separando la educación de la realidad social (MILENIO, 2017).

\section{Autonomía universitaria vs autonomía educativa}

Por supuesto que en el sistema de educación superior mexicano existe la autonomía universitaria, esta se refiere a "las relaciones de la universidad con instancias de decisión externas a ella. La autonomía es considerada un valor deseable por y para las universidades como condición para el pensamiento libre sin restricciones. Se acepta que el fundamento y reconocimiento legal de esa autonomía radica en que sin dicho atributo una universidad no podría cumplir de la mejor forma sus funciones de docencia, investigación y difusión de la cultura". Por el contrario, en este espacio por autonomía educativa nos referiremos a la acción mediante la cual la comunidad involucra todos los recursos disponibles para educar a sus jóvenes. Así como el derecho a decidir sus criterios de admisión, gestión, administración y enseñanza y aprendizaje, en un ambiente que involucra los recursos emocionales, sociales, políticos y culturales disponibles. Creando una relación a veces tirante entre la 
educación formal, para de facto ejercer la autonomía educativa. En contraste con la perspectiva institucional, donde la autonomía educativa se refiere a la necesidad de impulsar el desarrollo de las habilidades de los estudiantes "al aprender de forma autónoma” (Gonzales y Marquez, 2020; Vera Pérez, 2013; Toro, 2004)

\section{PROCEDIMIENTOS METODOLOGICOS}

Este documento es la síntesis de diversos ejercicios etnográficos, fruto de una relación de 8 años y acercamientos con miembros fundadores y alumnos de la Preparatoria 55, la Universidad Revolución (RU) y la Red de Universidades Alternativas (RUA). En clave propia de la antropología política y una perspectiva educativa nos acercaremos a organizaciones sociales que crean y plantean objetivos y estrategias para ejercer la autonomía educativa. Entrevistas dirigidas, observación y observación participante me permitieron nombrar de esta manera las experiencias observadas desde el 2013. Este documento se redacta a dos meses de terminar el año 2021, algunas de las experiencias que aquí se describen se encuentran menos fortalecidas ante un confinamiento de 18 meses. La incertidumbre reinante no permite ofrecer resultados concluyentes, pero considerando que estas iniciativas han resistido sexenios y represión política, podemos pensar que se mantendrán ante condiciones extremas, como lo han hecho por décadas.

Lo que si podemos concluir es que no contamos con una propuesta metodológica definitiva para estudiar la resistencia. Pero lo que si podemos afirmar es que estos movimientos orgánicamente son polifónicos, o no lo son, así como este documento. Quien aquí escribe, no puede más que limitarse a dar forma y traducir estas experiencias para desconocer su existencia.

\section{4.- RESULTADOS Y DISCUSIÓN}

\section{Educación desde los márgenes}

Al unánime consenso sobre la función de la Universidad en América Latina, su centralidad en la producción y difusión del conocimiento, el monopolio de la ciencia y tecnología, se le suma una carga aún más pesada que es la formación de la persona, la cual es frecuentemente utilizada como sinónimo de construcción de ciudadanía (Castañuela, 2017; Capello, 2015; Navarrete, 2013, Luque y Rojas, 2011; Garello y Rinaudo, 2010). De tal suerte que los proyectos educativos que se realizan y gestionan fuera de la institucionalidad, son invisibilizados o reducidos al ámbito de los llamados "saberes". 
Conocimiento de segundo orden, el cual tiene un valor moral pero no político ni científico. Curiosamente en los márgenes de la ciudad y la institucionalidad se han creado espacios de excepción, donde se configuran los recursos económicos, morales, culturales existentes, para dar a luz proyectos que han tomado en sus propias manos la educación de los miembros de su comunidad.

Resisten también a la cooptación y coorporativización de líderes y movilizaciones surgidas en la sociedad civil, cuestionando frontalmente el control de organismos como la SEP. Como ejemplo, podemos mencionar al Consejo de Transformación Educativa, creado hace 10 años, que reúne académicos y científicos sociales que analizan alternativas educativas y creando redes nacionales e internacionales de reflexión y dialogo. Otra importante iniciativa es la Red de Universidades Alternativas, la RUA, creada hace 4 años. Inspirada por los trabajos de la Red Latinoamericana de Intercambios de Saberes. Ninguna de las organizaciones arriba mencionadas está constituida como Asociaciones Civiles, el principio que los vincula es la solidaridad y cooperación. Esta es también la lógica de funcionamiento de la RUA, creada con la intención de promover el intercambio entre sus estudiantes, para que pudieran conocerse, aprender de otros modelos, que tuvieran donde realizar sus prácticas.

Entre las universidades fundadoras se encontraban la UR y la Universidad del Pueblo Trabajador de Neza, que ya no esta en la red. Comenzaron con 8 escuelas miembros, después fueron12, actualmente se cuentan hasta 45 miembros. Todas tienen perfiles e historias particulares, pero pueden de forma genérica agruparse entre universidades urbanas creadas en zonas con un nivel altísimo de marginalidad y violencia, que se enfocan en temas de desarrollo local sustentable, como la UR de Chicoloapan. Universidades cooperativistas, como la Universidad Cooperativa de Ecatepec. Las escuelas campesinas que son alrededor de 100, creadas hace 21 años. Universidades campesinas Indígenas. Universidades Interculturales como la del Estado de Sinaloa, de reciente reconocimiento. La escuela campesina indígena de Sonora. La UCIRED, Universidad campesina en RED. Universidad sustentable de Taranco. La Universidad del pueblo de Oaxaca, que agrupa entre 15 y 20 universidades. CEDECH en Cuernavaca. La Universidad José Revueltas o la Universidad Indígena de Chimalhuacán en el Estado de México. La Alianza Global de Ecoversidades. La más nueva será la Universidad Paulo Freire en Juan N. Méndez en Puebla, se acaba de anunciar su creación. Sus formas de financiamiento, sus propuestas pedagógicas y programas de estudio también son diferentes, pero se desenvuelven entre 7 y 8 áreas de conocimiento, que van desde desarrollo local sustentable, pasando por comunitarismo, cooperativismo, salud comunitaria, hasta el trabajo de la tierra. 
Las caracteriza su dinamismo, entrada y salida de miembros, sus alianzas políticas que ocasionalmente son instrumentales y determinadas por el contexto político reinante. Son siempre activas, enfocan su energía en la creación de ejercicios educativos que van de la mano con la cultura local, de salud, proyectos económicos o políticos. Encuentran fundamento en el modelo de educación popular (Bruno Jofre, 2016). Sus fuentes de financiamiento son variadas, algunas reciben "apoyo" del municipio, coyunturalmente de algún partido político o de instituciones estatales, federales o de nadie. Pero fundamentalmente funcionan con cuotas o cooperaciones de los alumnos y el trabajo voluntario de la comunidad. Su fuerza es el trabajo solidario de los profesores y directivos de las escuelas, de los padres y mesas directivas, que colaboran en la construcción de las instalaciones a través de faenas, manovuelta, tequio y todos los nombres que recibe el trabajo voluntario en sus contextos culturales.

\section{Ley General de Educación Superior 2021 ¿Izquierda de estado?}

Después de la toma de posesión de Andrés Manuel López Obrador, el entonces titular de la SEP, Esteban Moctezuma Barragán dio a conocer las transformaciones que se suscitarían en materia educativa. El 03 de diciembre, el nuevo secretario Otto Granados anunció la creación de infraestructura para ofrecer educación en zonas marginales e indígenas. El 09 del mismo mes anunció la paulatina descentralización de la SEP. En esta oleada de cambios, el 20 de abril del 2021, en el Diario Oficial de la Federación se publicó el decreto por el que se expide la Ley General de Educación Superior (LGES) y se abroga la Ley para la Coordinación de la Educación Superior. Estableciendo, según la lógica de gobierno, las bases para dar cumplimiento a la obligación del Estado de garantizar la educación superior como un derecho (DOF 20 de abril de 2021). En su artículo segundo dicta que a las universidades e instituciones de educación superior a las que la ley otorgue autonomía contarán con todas las facultades y garantías institucionales que se establecen en la fracción VII del artículo 3o. de la Constitución Política. Rigiéndose por sus respectivas leyes orgánicas en tanto que sean compatibles con la ley. Como antecedente en el 2019 se modificó el artículo 3ero constitucional para establecer la obligatoriedad de la educación superior. El mismo año se publicó también el estatuto del Organismo coordinador de las Universidades para el Bienestar, Benito Juárez García. Actualmente se cuenta ya con 140 planteles establecidas en zonas de marginalidad y alta marginalidad.

Estos cambios profundos y estructurales, positivos desde una visión de estado, han representado un esfuerzo enorme por fortalecer la centralidad gubernamental de la educación superior. En octubre del año 2021 miembros de la RUA, sostuvieron una reunión con representantes de la Dirección General de Acreditación, Incorporación y Revalidación (DGAIR), la cual depende de la SEP. En esta se les 
comunico que se aplazaría una vez más la solicitud de las universidades alternativas por obtener su certificado de Reconocimiento de Validez Oficial de Estudios (REVOE), con esto se les coloca nuevamente en la marginalidad. Aparentemente la promulgación de la LGDS busca contrarrestar la oleada de reformas educativas implementadas en las décadas de los 80 y 90 en América Latina, que entregaron la educación a la iniciativa privada (Di Gropello, 1999), pero deja fuera iniciativas que ya lo venían haciendo desde hace casi un cuarto de siglo.

Otro dilema importante que enfrentan estos proyectos es que a pesar de que muchas, muchos de ellos apoyaron la campaña política de la actual administración, no han recibido el apoyo y reconocimiento que esperaban. Una acción política muy negativa para ellos fue la creación de las Universidades del Bienestar Benito Juárez. Y más aún la publicación de la LGDS, que las sitúa en la ilegalidad, que no las incluye ni las reconoce. Este tema es inconcluso, pues a 3 años de que termine este sexenio, aun no se sabe cómo se perfilará la relación con el Estado.

\section{Frente a la centralización de la educación: trabajo en red}

Ante el intento por centralizar la educación, el fortalecimiento de las redes es la solución. Es decir, la creación de más universidades y planteles no resuelve el problema educativo, ni reduce la brecha entre la formación universitaria y la realidad social. Por el contrario, la propuesta educativa de la RUA, surge y se alimenta de los movimientos obreros, vecinales y de la izquierda no partidaria. Son, en palabras de uno de sus fundadores, efectos tardíos del zapatismo, ante una inercia estatal que se sigue reproduciendo con todos sus vicios. La red ha buscado y ha encontrado redes similares en Latinoamérica. Durante el confinamiento, se han realizado encuentros en diversas plataformas virtuales. Se siguen compartiendo ideas, trabajo, experiencias. En el caso concreto de la RUA, así como hay intereses que las unen, las separan las diversas practicas pedagógicas y andragogías aplicadas. Las divide su origen político y sus perspectivas sobre la política partidaria, incluso sobre su visión de Estado o la necesidad de mantenerse al margen de él. En palabras de su fundador "Nos unen nuestras diferencias y nos separan nuestras cosas comunes" (López 47 entrevista 24 de octubre 2021)

Desde el inicio de este sexenio la red ha mantenido el dialogo con la SEP en busca de su reconocimiento, pero la respuesta que han obtenido es que esperen, que no se preocupen, que las Universidades del Bienestar no los van a absorber. Pero el hecho de que las hayan construido al lado o en el mismo poblado donde ya existían universidades alternativas es políticamente muy negativo. Solo pueden compararse con las practicas anti insurgentes en Chiapas, en las cuales se les inyectan recursos a las comunidades priistas situadas a un lado de las comunidades en resistencia zapatista. Quizá, como 
en el sureste mexicano se apuesta al olvido. Pero ante el olvido, la creación de organizaciones en resistencia, en este caso de resistencia educativa. Como perfectamente lo define el Ingeniero Víctor López, maestro de la Universidad Revolución, las universidades miembros de la red son las autodefensas educativas.

Para los que se rigen bajo la lógica del sistema de partidos es difícil entender, que, con un partido de izquierda en el gobierno, organizaciones civiles que alguna vez lo apoyaron reacciones de esta manera. Bajo una lectura política sistémica, la existencia de organizaciones o redes como estas seria innecesaria, toda vez que han pasado por el gobierno todas las alternativas políticas, con todas las alianzas políticas inimaginables que un país como México puede ofrecer. Vemos entonces que el Estado en su dimensión gobierno no comprende la dimensión de la emergencia educativa que vivimos, quizá porque ya se convirtió en la normalidad. La experiencia acumulada por las universidades de la red podría aportar bastante en materia educativa, pues saben existir ante la adversidad, las soluciones que han creado para educar a sus jóvenes surgen desde dentro, con conocimiento real de lo que necesitan.

\section{Hacia la autonomía educativa}

Las políticas educativas lanzadas por la actual administración y su indecisión sobre otorgar el reconocimiento oficial de la SEP, vía la DGAIR a los miembros de la RUA, han impulsado y acelerado las respuestas de los miembros de la RUA. La cual, por supuesto, es siempre innovadora, creativa y propositiva. El 12 y 13 de noviembre del presente año, se celebró en línea el Multicongreso, al cual asistieron miembros de aproximadamente 50 organizaciones. Como acto de apertura se leyó el Manifesto que anunció la creación del Colegio de Certificación, Reconocimiento, Acreditación y Validez de Estudios y Saberes (CCRAVES). Acción inédita en la historia de la educación, ya que se pronunciaron por la formación de opciones alternativas no centralizadas o institucionalizadas que acrediten y dignifiquen la diversidad de procesos de enseñanza- aprendizaje, que se realizan en el país, marginados por la ley establecida en el marco de las actuales políticas educativas.

Dicho Colegio estará conformado por El Consejo de Transformación Educativa, El Movimiento Nacional de Escuelas Campesinas y la Red de Universidades Alternativas. Reconociendo que la emergencia ambiental, educativa y de seguridad pública deberían ser temas de primer orden y no pueden ser conferidos, para su solución, a unos cuantos que responden a intereses que no son los de las comunidades. En palabras de algunos de los ponentes, de este gobierno "quedaron las buenas intenciones, quedo la esperanza". Sus decisiones en materia educativa son leídas como "la 
continuación de la mercantilización de la educación”. Y ante este panorama, de emergencia ambiental, social y educativa, las universidades y organizaciones aquí agrupadas en una red de redes apuestan a su auto-reconocimiento, a su autovalidación. Decisión valida toda vez que ellos son los especialistas en educación popular y comunitaria, lo han practicado por más de 3 décadas, son los especialistas culturales, médicos tradicionales, campesinos, trabajadores de la tierra y, sobre todo, surgen de la realidad que la academia aspira a estudiar y mejorar.

\section{CONCLUSIONES:}

En este espacio presentamos proyectos que apuestan por la autonomía educativa, misma que se refiere a las prácticas de enseñanza aprendizaje fuera y dentro del aula, pero no se limita a ello. Se extiende a la autonomía curricular, a la autogestión del conocimiento, de recursos, a la innovación de sus planes y programas, a la incursión en campos de conocimiento que el positivismo académico no incluye. Esta autonomía busca la solución de problemas prácticos, a mejorar el entorno comunitario y a restablecer el tejido social e incluso a construir otros tejidos que ni siquiera sabíamos que podrían existir. Ante una crisis educativa sin precedentes, se vuelve urgente voltear la mirada a las propuestas andragógicas y pedagógicas de universidades que conforman la RUA y ahora el CCRAVES. Propuestas que sostienen que la persona es indivisible del alumno, y su crecimiento solo será posible cuando vaya de la mano del desarrollo integral de la comunidad a la cual este pertenece.

Estamos conscientes de que este tema desata muchas otras aristas por investigar y profundizar, pero entre ellas rescatamos en este espacio 3 ámbitos que vinculan la acción del gobierno actual la 4T en materia educativa, las propuestas autónomas de educación media y superior y la crisis educativa. Sobre este tema resultan muy interesantes muchos puntos, pero aun más cuando se desatan muchas controversias y sus protagonistas enfrentan nuevos dilemas, por ejemplo, que las propuestas de la RUA no eran nuevas para la actual administración. Los miembros de la 4T no son ajenos a estos procesos, se reunieron con ellos, los conocen, han compartido luchas y espacios. Y los miembros de la RUA enfrentan el dilema de caminar de nuevo solos y olvidar la posibilidad de ser incluidos en este proyecto de gobierno; superando, como señalaron en el multi-congreso del mes de noviembre, el estatismo, el presidencialismo, las ciegas políticas de Estado. Ante una crisis educativa agudizada parece que nos enfrentamos a políticas publicas que no logran la intermediación y al contrario promueve una educación sin experimentación, no significativa ni transversal. Aun falta por saber que pasara en los siguientes 3 años de gobierno, pero desde la comunidad se han dado los primeros pasos para abatir la 
crisis educativa, para extender el significado de educación y crear instancias no gubernamentales que amplíen el sentido de educar.

\section{REFERENCIAS}

Aguirre Rojas, C. A. (2017). Mapa de los movimientos antisistémicos de América Latina. Theomai, (36), 128-147. https://www.redalyc.org//124/12453261009.pdf

Arendt, H., Romero, J. M., \& Bayón Cerdán, J. (1993). La crisis de la educación. Sugerencias y Frustraciones Cuaderno Gris. (s/n) 38-53. https://repositorio.uam.es/bitstream/handle/10486/260/22409_La\%20crisis\%20de\%20la\%20educaci\%C3\%B3n.p df

Batta Fonseca, V. (2008). Altermundismo: ¿sociedad civil global o nuevo movimiento antisistémico?. Norteamérica, 3(2), 159-194. http://www.scielo.org.mx/pdf/namerica/v3n2/v3n2a8.pdf

Bruno-Jofré, R. (2016). Educación popular en América Latina durante la década de los setenta y ochenta: una cartografía de sus significados políticos y pedagógicos. Foro de Educación, 14(20), 429-451. http://dx.doi.org/10.14516/fde.2016.014.020.021

Caicedo, K. V., \& Zuluaga, Y. E. L. (2015). Educación en emergencias o emergencias en educación. Plumilla Educativa, 16(2), 324-341.

https://doi.org/10.30554/plumillaedu.16.1614.2015

Cappello, H. M. (2015). La identidad universitaria. La construcción del concepto. Revista Internacional de Ciencias Sociales y Humanidades, SOCIOTAM, 25(2), 33-53. https://www.redalyc.org/pdf/654/65452536003.pdf

Castañuela Sánchez, B. G. (octubre-diciembre 2017). La universidad y su función social. CienciAcierta Revista científica, tecnológica y humanística (48), 1-13. http://www.cienciacierta.uadec.mx/2016/12/14/la-universidad-y-sufuncion-social/

Cazales, Z. N. (2013). La universidad como espacio de formación profesional y constructora de identidades. Universidades, (57), 5-16. https://www.redalyc.org/pdf/373/37331246003.pdf

Cristi, I. M. (2018). Autogestión Compleja en un Movimiento Urbano Popular: Prefigurando el Buen Vivir en una lucha contra, sin y desde el Estado-Mercado. [Resumen de la ponencia] Congreso de la RED PILARES 2018, Santiago de Chile. https://bit.ly/3qFiFoX

Decreto de Ley General de Educación Superior y se abroga la Ley para la Coordinación de la Educación Superior. 20 de abril de 2021. Diario Oficial de la Federación. CDMX.

De la Parra, M. L. (2014). La UNAM y la Facultad de Economía en la historia de México. Economía Informa, 386, 64-76. http://www.economia.unam.mx/assets/pdfs/econinfo/386/05Lopezdelaparra.pdf

De la Torre Jesús. (25-08-2017). La educación en la emergencia educativa. Milenio. https://www.milenio.com/opinion/jesus-de-la-torre-pbro/areopago/la-educacion-en-la-emergencia-educativa

De La Torre L, C. F. (2016). El trabajo misional de fray Pedro de Gante en los inicios de la Nueva España. Fronteras de la Historia, 21(1), 90-116. https://www.redalyc.org/pdf/833/83346866004.pdf

Díaz Miranda, Delgado Celis G.A., \&, Yael Zaira. (Coords). (2021). Emergencia sanitaria, emergencia educativa . Educación cultura Libre. UNAM. https://chat.iztacala.unam.mx/index.php/libro/emergencia-sanitariaemergencia-educativa

Forbes Staff. (2021, 22 junio). La población mexicana usuaria de internet aumentó $72 \%$ en 2020. Forbes México. https://www.forbes.com.mx/la-poblacion-mexicana-usuaria-de-internet-aumento-72-en-2020/

Entreculturas ( s/f). ¿Qué es la educación en emergencia? https://escuela.entreculturas.org/que-es-educacion-enemergencia/ 
Garello, M. V., Rinaudo, M. C., \& Donólo, D. (2010). Construcción del conocimiento y desarrollo académico en la universidad. Revista de la educación superior, 39(154), 91-107.

http://www.scielo.org.mx/scielo.php?script=sci_arttext\&pid=S0185-27602010000200005

García Vásquez, E. (2015). La discriminación por discapacidad como tema emergente en el contexto de los movimientos sociales contemporáneos. Revista de la Facultad de Medicina, 63(3Sup), 155-160. https://doi.org/10.15446/revfacmed.v63n3sup.50571

González Meyer, Raúl., \& Márquez, F. (12-2020). Universidad: crítica para la autonomía, vocación pública y saberes otros. Atenea (Concepción), (522), 59-81. http://dx.doi.org/10.29393/at522-96ucrg20096

Humanitarian Response (8-11-2021). Educación en emergencia. https://www.humanitarianresponse.info/es

Luque, T. S., \& Rojas, C. C. (noviembre 2011). Influencia de la universidad en la construcción de la sociedad del conocimiento. Contribuciones a las Ciencias Sociales. (14) https://www.eumed.net/rev/cccss/15/slcr.html

Marsiske, R. (2006). La Universidad de México: historia y desarrollo. Revista Historia de la Educación Latinoamericana (8) 11-34. https://www.redalyc.org/pdf/869/86900802.pdf

Martínez Paris. (2-12-2012). Termina el paro en la UACM. Animal Político. https://www.animalpolitico.com/2012/11/termina-el-paro-en-la-uacm/

Nájera, I. L. (2020). Pugnas en torno a la gratuidad: los huelguistas del movimiento estudiantil 1999-2000 en la UNAM. Clivajes. Revista de Ciencias Sociales, (13), 98. https://doi.org/10.25009/clivajes-rcs.v0i13.2650

Ornelas, C. (2012). Educación, colonización y rebeldía: la herencia del pacto Calderón-Gordillo. Siglo XXI.

Peralta, M. T. (2019). Economías solidarias y movimientos sociales La opinión de los actores. En Memoria de trabajos extensos del III Congreso Mexicano de Sociología, 137-151. https://bit.ly/3ozNpos

Rodriguez Araujo, O. (2000) El conflicto en la UNAM (1999-2000). Dos concepciones sobre la universidad pública. En Rodriguez Araujo, O. (coord.) El conflicto en la UNAM (1999-2000) 11-67. El Caballito.

Rodríguez Gómez, R. (Coord.). (2013). El siglo de la UNAM: Vertientes ideológicas y políticas del cambio institucional. Perfiles educativos. UNAM. https://www.ses.unam.mx/integrantes/uploadfile/rrodriguez/Rodriguez2013_SigloUNAM.pdf

Rodríguez Gómez, R. (2015). El proyecto educativo SEP-SNTE y la prueba ENLACE. Revista mexicana de investigación educativa, 20(64), 309-324. http://www.scielo.org.mx/scielo.php?script=sci_arttext\&pid=S140566662015000100015

Ruiz Muñoz, M., \& Luna Guzmán, A. (2017). El derecho a la educación en el nivel medio superior en México. Revista latinoamericana de educación inclusiva, 11(2), 73-90. http://dx.doi.org/10.4067/S0718-73782017000200006

Toro, J. R. (2004). La autonomía, el propósito de la educación. Revista de estudios sociales, (19), 119-124. https://journals.openedition.org/revestudsoc/24435

Vera Pérez, B. L. (2013). La autonomía educativa ante los nuevos paradigmas, un criterio a seguir en la educación continua. Ciencia Huasteca Boletín Científico de la Escuela Superior de Huejutla, 1(1). https://repository.uaeh.edu.mx/revistas/index.php/huejutla/article/download/1006/4828?inline=1

Viáfara López, C. A. (2017). Movilidad social intergeneracional de acuerdo al color de la piel en Colombia. sociedad y economía, (33), 263-287. https://www.redalyc.org/pdf/996/99654715011.pdf

Zablotsky Edgardo. (2-12-2020). Emergencia educativa es la única alternativa. INFOBAE https://www.infobae.com/opinion/2020/11/26/emergencia-educativa-es-la-unica-alternativa/

Zibechi, R. (2014). Liberar el mundo nuevo que late en el corazón de los movimientos. Revista Kavilando, 6(1), 7-14. http://www.kavilando.org/revista/index.php/kavilando/article/view/71 